\title{
Use of a New Knee Prosthesis as an Articulating Spacer in Two-Stage Revision of Infected Total Knee Arthroplasty
}

\author{
Rasmus Juul, $\mathrm{MD}^{1}$, Jesper Fabrin, $\mathrm{MD}^{1}$, Klaus Poulsen, $\mathrm{MD}^{1}$, and Henrik Morville Schroder, $\mathrm{MD}^{2}$ \\ ${ }^{1}$ Department of Orthopaedic Surgery, Zealand University Hospital, Koge; ${ }^{2}$ Department of Orthopaedic Surgery, Naestved Hospital, Naestved, Denmark
}

\begin{abstract}
Purpose: To report our experience with two-stage revision using a new femoral component (NFC) spacer (Depuy Synthes) as an articulating spacer. Materials and Methods: In this retrospective study, we reviewed 22 two-stage revisions that were performed using an NFC spacer in 22 patients suspected of having an infected total knee arthroplasty (TKA) from December 2010 to March 2013. The result was considered successful when eradication of infection was achieved using only one NFC spacer.

Results: The average time from primary TKA to the first stage procedure was 29.1 months and the average time from the first stage procedure until the final second stage procedure was 12.7 weeks. The average range of motion increased from $82^{\circ}$ preoperatively to $104^{\circ}$ postoperatively. The American Knee Society Knee score increased from 29.3 points to 66 points. The Function score increased from 29.5 points to 64 points. Four cases were reinfected after two-stage revision. The mean follow-up was 37.6 months.

Conclusions: The new articulating spacer showed promising short-term results both with regard to eradication of infection and functional improvement.
\end{abstract}

Keywords: Knee, Arthroplasty, Infection, Revision, Two-stage, Articulating spacer, New prosthesis

\section{Introduction}

The incidence of infection after primary total knee arthroplasty (TKA) has been reported to be $1 \%$ with an increase to $5 \%$ in patients with revision $\mathrm{TKA}^{1,2)}$. For many years, the gold standard for treatment of chronic periprosthetic joint infection (PJI) has been two-stage revision. Static cement antibiotic spacers are well known to be an effective treatment for infection in $\mathrm{TKA}^{3,4)}$. Static cement spacers provide local antibiotic therapy while maintain-

Received February 18, 2015; Revised (1st) June 17, 2015;

(2nd) October 11, 2015; (3rd) February 3, 2016; (4th) February 17, 2016

Accepted March 4, 2016

Correspondence to: Rasmus Juul, MD

Department of Orthopaedic Surgery, Zealand University Hospital,

Lykkebækvej 1, Koge 4600, Denmark

Tel: +45-4076-4590, Fax: +45-56-63-15-39

E-mail: rasmusjuul@dadlnet.dk

This is an Open Access article distributed under the terms of the Creative Commons Attribution Non-Commercial License (http://creativecommons.org/licenses/by-nc/4.0/) which permits unrestricted non-commercial use, distribution, and reproduction in any medium, provided the original work is properly cited. ing soft-tissue tension until the second stage procedure. With the use of static spacers, the leg is braced with minimal motion. This may lead to stiffness of the knee and problems such as bone loss, pain, scarring, and instability,

To overcome these problems, articulating spacers have been developed. Articulating spacers have the advantage that they allow weight bearing and maintain joint movement, thereby improving patient's satisfaction, reducing bone loss, facilitating the secondstage procedure, and resulting in a greater range of motion (ROM) in the end ${ }^{7}$. Several studies have shown articulating spacers have the same reinfection rates as static spacers while, to some extent, preserving the function of the knee in between stages ${ }^{8,9}$.

A number of methods using articulating spacers have been described. Two types of cement-on-cement articulating spacer have been presented: either those that can be made during operation using antibiotic-loaded cement and silicon mold components or prefabricated and commercially available cement spacers ${ }^{9,10)}$. Articulating spacers can also be made reusing the original autoclaved femoral component with a new tibial polyethylene insert or using a new femoral component and a new tibial polyethylene 
insert (NFC spacer; PFC Sigma, Depuy Synthes, Warsaw, IN, USA). Both techniques have shown good results with regard to infection control and $\mathrm{ROM}^{11)}$.

The aim of this paper is to report our experience with an NFC spacer in terms of both eradication of infection and functional improvement.

\section{Materials and Methods}

From December 2010 to March 2013 (28 months), a consecutive series of 32 two-stage revisions using NFC spacers were performed in 31 patients suspected of having a chronic infected TKA. We only included patients with a primary TKA who had not received any prior two-stage revision for PJI. In all cases, patient's medical records and radiographs were reviewed retrospectively after approval was obtained by the Danish data protection agency.

The diagnostic criteria for PJI are difficult and numerous definitions have been proposed. In our study, patients were diagnosed with PJI based on clinical suspicion (pain, swelling, redness, or warmth), radiography, white blood cell count (WBC), and Creactive protein (CRP) level or microbiologic examination of preoperative joint fluid, although knee aspiration was not performed routinely. The diagnosis was verified from microbiologic examination of joint tissue at the time of first-stage surgery. In general, a positive culture result of 2 or more than 5 intraoperative cultures was considered an infection ${ }^{12}$. A PJI may be present although none or only one out of 5 intraoperative cultures is positive. Several other factors such as presence of purulence in the affected joint, elevated synovial leukocyte count, and elevated CRP concentration also need to be considered in order to determine the presence of PJI. Retrospectively, all our cases were confirmed to have PJI according to the universally adopted new definition for $\mathrm{PJI}^{13)}$ used to ensure consistency in definition in this study.

Every patient underwent a 2-stage exchange with an NFC spacer. At first stage, the prosthetic components and bone cement were removed. Thorough debridement of the infected and devitalized tissue and bone was performed, which was followed by pulsating saline lavage. Five intraoperative cultures were taken from inflamed synovial tissue or bone-cement membrane before the administration of intravenous antibiotics ${ }^{12}$. NFC spacers were cemented with Refobacin Revision Bone Cement (Biomet, Valance, France). A sachet of $40 \mathrm{~g}$ Refobacin Revison Bone Cement contains $1 \mathrm{~g}$ gentamicin and $1 \mathrm{~g}$ clindamycin (Fig. 1). The cement was in a late doughy stage so it would not adhere well to the bone, thereby allowing removal without any major bone loss at the time of second stage procedure.

According to a standard protocol, patients who were considered positive for PJI received a minimum of six-week antibiotic treatment including two postoperative weeks of intravenous administration and oral administration thereafter. Every patient received antibiotic treatment targeting the specific microorganism after consultation with a microbiologist.

Patients were encouraged to mobilize with full weight bearing and perform ROM exercises as tolerated immediately after the first stage procedure with the help of a physiotherapist.

The second stage procedure in the revision TKA using the PFC Sigma TC3 Knee System (Depuy Synthes) was scheduled minimum 2 weeks after the first stage procedure without antibiotic administration. There was no rise in the inflammatory blood markers combined with the clinical appearance of the soft tissue (Fig. 2). No joint aspiration was performed in between stages.

Of the 32 cases identified, only 22 were included in the study.

One patient was excluded due to prior two-stage surgery with a static cement spacer immediately before the two-stage revision using an NFC spacer and two patients died from unrelated medical reasons and were lost to follow-up.

One patient with a bilateral NFC spacer had a dislocation tendency in the left knee and a chronic resistant infection in the left Charcot foot. An above knee amputation of the left knee with an NFC spacer was required and thus this patient was excluded from the analysis.

Another 6 patients who had two-stage revisions using NFC spacers were excluded for the following reasons. First, they had negative tissue cultures in all 5 samples collected during the first
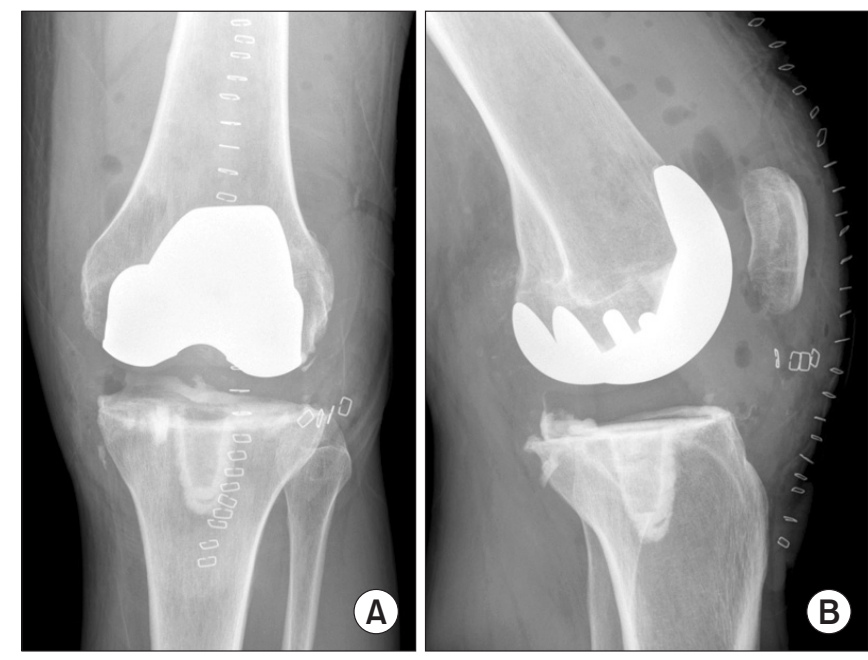

Fig. 1. Anteroposterior (A) and lateral (B) radiographs of the knee with an new femoral component spacer on the first postoperative day. 


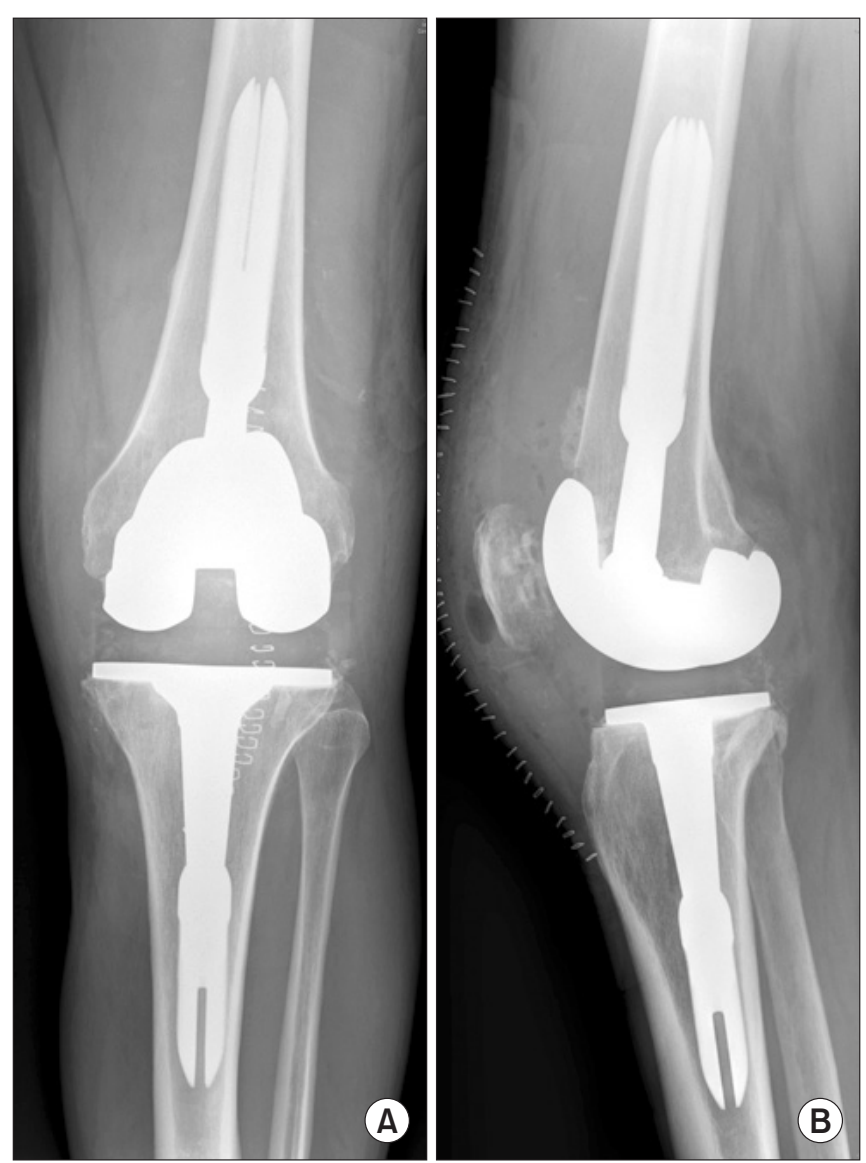

Fig. 2. Anteroposterior (A) and lateral (B) radiographs following the second stage procedure in revision total knee arthroplasty.

stage of the surgery despite clinical signs of infection prior revision surgery such as pain, swelling and increased CRP. Second, they did not meet the other criteria for PJI according to new definition for $\mathrm{PJI}^{13)}$. Therefore, they only received antibiotic treatment ranging from 4-13 days postoperatively until negative tissue cultures were obtained. They all had a successful second stage procedure within 4-12 weeks after the first stage surgery when soft tissue allowed the procedure. No reinfection was observed in these 6 cases.

All patients were followed up with an outpatient visit at 6 weeks and 1 year after surgery. From almost all patients, American Knee Society knee score (AKSS), function score, and ROM were obtained prior to the first stage surgery and one year after the second stage surgery, which were registered in the Danish Knee Arthroplasty Registry. All patients were reviewed at "e-journal", which is an internet-based medical journal for physicians where all contacts with the Danish Health Care System is stored, to ensure that no patient had any additional knee surgery at other institutions after the second stage procedure at our institution.
The result after the 2-stage revision using an NFC spacer was considered successful when eradication of infection was achieved using only one NFC spacer.

\section{Results}

The 22 cases meeting the diagnostic criteria for PJI and included in the follow-up consisted of 12 females 10 males with an average age of 65.7 years (range, 49 to 82 years). All had a twostage revision using an NFC spacer with a mean follow-up of 37.6 months (range, 25 to 52 months).

Cases 1-15 (Table 1) had 2 or more positive tissue cultures out 5 samples except one patient (case 5 ) that had negative test results in all 5 tissue cultures but presented obvious purulent material in the knee joint at the first stage surgery along with other signs of PJI. All 15 cases, therefore, received our standard antibiotic regimen with a minimum 6-week antibiotic treatment before the second stage procedure. Eradication of infection was achieved using only one NFC spacer in all 15 cases and no reinfection was observed.

Cases 16-18 were primarily not considered positive for PJI because none or only one out of 5 intraoperative cultures was positive. Therefore, they only received antibiotic treatment for a few days postoperatively until negative tissue cultures were obtained. Retrospectively, PJI was confirmed by the new definition for $\mathrm{PJI}^{13)}$ in the patients, and, therefore, they should have received a standard antibiotic regimen for a minimum of six weeks before the second stage procedure. However, they all had a successful second stage procedure and no reinfection was observed in these 3 cases.

Cases 19-22 were positive for PJI with 2 or more positive tissue cultures and had a recurrence of infection after their first second stage procedure. Cases 19 and 20 were treated with multiple spacers (more than 2) at our institution and still remained infected, and consequently they were referred to other institutions for further treatment and, therefore, they were lost to follow-up. Case 21 was infected with coagulase-negative staphylococcus and had a successful two-stage procedure and no reinfection was observed for several years. But due to urine retention, the patient had numerous urological procedures and therefore became reinfected with Escherichia coli as a result of hematogenous dissemination. The patient has undergone two-stage revision for a second time and just recently received a new revision TKA. Case 22 became reinfected with the same species (coagulase-negative staphylococcus) and was treated with two-stage revision for a second time but has shown no sign of reinfection after receiving a second re- 


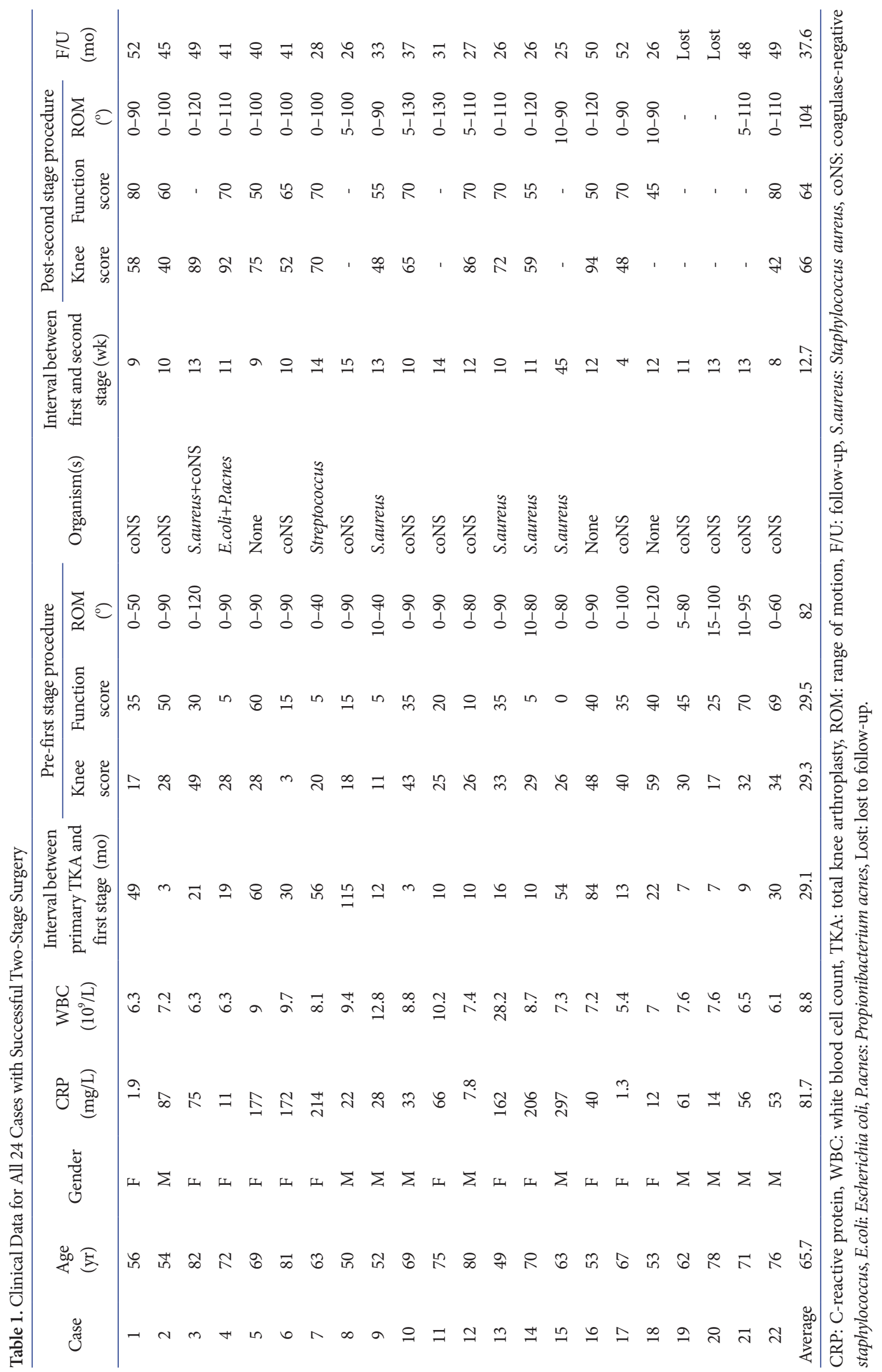


vision TKA more than a year ago.

The average time from primary TKA to the first stage procedure was 29.1 months (range, 3 to 115 months). The average value of CRP was $81.7 \mathrm{mg} / \mathrm{L}$ (normal reference $<8 \mathrm{mg} / \mathrm{L}$ ) before the first stage surgery and the average value of WBC was $8.8 \times 10^{9} / \mathrm{L}$ (normal reference $3,5-8,8 \times 10^{9} / \mathrm{L}$ ) before the first stage surgery. The AKSS was 29.3 (range, 3 to 59), function score was 29.5 (range, 0 to 70 ), and the average $\mathrm{ROM}$ was $82^{\circ}$ (range, $40^{\circ}$ to $120^{\circ}$ ) before the first stage surgery.

The average time from the first stage procedure to the final second stage procedure was 12.7 weeks (range, 4 to 45 weeks). At the last scheduled follow-up in our outpatient clinic 1 year postoperatively from the second stage surgery, the AKSS had improved to 66 (range, 40 to 94 ) and the function score was 64 (range, 45 to 80 ). The average ROM had improved to $104^{\circ}$ (range, $80^{\circ}$ to $130^{\circ}$ ).

Successful eradication of infection was achieved in 18 (82\%) out of the 22 cases that were positive for PJI using only one NFC spacer.

\section{Discussion}

Periprosthetic infection remains a devastating and challenging complication of TKA for both the patient and the surgeon. Twostage revision with an articulating spacer has shown good results in terms of infection control ${ }^{7,9)}$, but very few studies have been reported on the use of an NFC spacer as an articulating spacer in two-stage revision of chronic PJI in TKA ${ }^{11,14,15)}$. The purpose of the present study was to present our short-term results using an NFC spacer as an articulating spacer.

Successful eradication of infection was achieved in 18 (82\%) of 22 cases using only one NFC spacer and this is comparable to other studies describing the use of an NFC spacer ${ }^{11}$; however, none of our patients needed long-term antibiotic suppression after the second stage procedure.

Eradication rate of $82 \%$ is comparable to that in the study by Park et al. ${ }^{3)}$ and Fehring et al. ${ }^{6}$ where the use of static cement spacers in two-stage revision resulted in the eradication rate of $85 \%$ and $88 \%$, respectively. Although our results in terms of the eradication of infection seems to be no better than that in the knees with static spacers, early ambulation immediately after the first stage surgery, which is possible due to the use of an NFC spacer, helps patients to perform activities of daily living, thereby improving patient's satisfaction and compliance.

Studies comparing articulating spacers with static spacers report greater postoperative function scores and ROM in the knees with articulating spacers ${ }^{9,16)}$. Brunnekreef et al. ${ }^{16)}$ reported significantly greater postoperative ROM and faster recovery in the group with articulating spacers. Limitations of our study include the relatively small study population and the lack of comparison with a control group. However, our study demonstrated good functional results of two-stage revision with an NFC spacer: ROM increased from $82^{\circ}$ to $104^{\circ}$ and function score increased from 29.5 to 64 postoperatively.

Previous studies have described good results with the use of an autoclaved femoral component as an articulating spacer ${ }^{17,18)}$. Compared to an NFC spacer, the autoclaved original femoral component is cost-effective ${ }^{11)}$; however, we are concerned about reusing an implant designed for single use only ${ }^{19)}$.

At our institution, a NFC spacer costs $\$ 573$ and \$150, respectively. Therefore, the total price for an NFC spacer is $\$ 723$, which is less than the price of our prefabricated articulating cement spacer (Spacer K-with gentamicin, Tecres, Verona, Italy), which costs $\$ 1,055$ albeit we are aware that regional differences in pricing can occur.

Another benefit of the NFC spacer is that it gives the surgeon the opportunity to balance the knee by adjusting the thickness of the polyethylene insert compared to the prefabricated articulating cement spacers that often only come in a few sizes.

Our study demonstrated a significantly improvement in both AKSS and ROM in the knees with an NFC spacer. Thus, the present spacer model seems to be a safe device for the treatment of infection of the knee offering good functional results.

\section{Conclusions}

Short-term results from our study provide evidence that the use of a NFC spacer as an articulating spacer is effective in two-stage revision of chronic infected TKA with regard to eradication of infection and functional improvement.

\section{Conflict of Interest}

No potential conflict of interest relevant to this article was reported.

\section{References}

1. Blom AW, Brown J, Taylor AH, Pattison G, Whitehouse S, Bannister GC. Infection after total knee arthroplasty. J Bone Joint Surg Br. 2004;86:688-91.

2. Willis-Owen CA, Konyves A, Martin DK. Factors affecting the incidence of infection in hip and knee replacement: an 
analysis of 5277 cases. J Bone Joint Surg Br. 2010;92:1128-33.

3. Park SJ, Song EK, Seon JK, Yoon TR, Park GH. Comparison of static and mobile antibiotic-impregnated cement spacers for the treatment of infected total knee arthroplasty. Int Orthop. 2010;34:1181-6.

4. Silvestre A, Almeida F, Renovell P, Morante E, Lopez R. Revision of infected total knee arthroplasty: two-stage reimplantation using an antibiotic-impregnated static spacer. Clin Orthop Surg. 2013;5:180-7.

5. Calton TF, Fehring TK, Griffin WL. Bone loss associated with the use of spacer blocks in infected total knee arthroplasty. Clin Orthop Relat Res. 1997;(345):148-54.

6. Fehring TK, Odum S, Calton TF, Mason JB. Articulating versus static spacers in revision total knee arthroplasty for sepsis: the Ranawat Award. Clin Orthop Relat Res. 2000; (380):9-16.

7. Hsu YC, Cheng HC, Ng TP, Chiu KY. Antibiotic-loaded cement articulating spacer for 2-stage reimplantation in infected total knee arthroplasty: a simple and economic method. J Arthroplasty. 2007;22:1060-6.

8. Emerson RH Jr, Muncie M, Tarbox TR, Higgins LL. Comparison of a static with a mobile spacer in total knee infection. Clin Orthop Relat Res. 2002;(404):132-8.

9. Freeman MG, Fehring TK, Odum SM, Fehring K, Griffin WL, Mason JB. Functional advantage of articulating versus static spacers in 2-stage revision for total knee arthroplasty infection. J Arthroplasty. 2007;22:1116-21.

10. Pitto RP, Castelli CC, Ferrari R, Munro J. Pre-formed articulating knee spacer in two-stage revision for the infected total knee arthroplasty. Int Orthop. 2005;29:305-8.

11. Kalore NV, Maheshwari A, Sharma A, Cheng E, Gioe TJ. Is there a preferred articulating spacer technique for infected knee arthroplasty? A preliminary study. Clin Orthop Relat Res. 2012;470:228-35.

12. Kamme C, Lindberg L. Aerobic and anaerobic bacteria in deep infections after total hip arthroplasty: differential diagnosis between infectious and non-infectious loosening. Clin Orthop Relat Res. 1981;(154):201-7.

13. Parvizi J, Zmistowski B, Berbari EF, Bauer TW, Springer BD, Della Valle CJ, Garvin KL, Mont MA, Wongworawat MD, Zalavras CG. New definition for periprosthetic joint infection: from the Workgroup of the Musculoskeletal Infection Society. Clin Orthop Relat Res. 2011;469:2992-4.

14. Scott IR, Stockley I, Getty CJ. Exchange arthroplasty for infected knee replacements: a new two-stage method. J Bone Joint Surg Br. 1993;75:28-31.

15. Trezies A, Parish E, Dixon P, Cross M. The use of an articulating spacer in the management of infected total knee arthroplasties. J Arthroplasty. 2006;21:702-4.

16. Brunnekreef J, Hannink G, Malefijt Mde W. Recovery of knee mobility after a static or mobile spacer in total knee infection. Acta Orthop Belg. 2013;79:83-9.

17. Anderson JA, Sculco PK, Heitkemper S, Mayman DJ, Bostrom MP, Sculco TP. An articulating spacer to treat and mobilize patients with infected total knee arthroplasty. J Arthroplasty. 2009;24:631-5.

18. Kim YS, Bae KC, Cho CH, Lee KJ, Sohn ES, Kim BS. Twostage revision using a modified articulating spacer in infected total knee arthroplasty. Knee Surg Relat Res. 2013;25:180-5.

19. Pitto RP, Spika IA. Antibiotic-loaded bone cement spacers in two-stage management of infected total knee arthroplasty. Int Orthop. 2004;28:129-33. 